\title{
Engaging parents to research childhood interventions aimed at preventing common health problems
}

\author{
Theresa H.M. Kim MSc PhD, Erika Tavares MSc PhD, Catherine S. Birken MD MSc
}

Cite as: CMAJ 2018;190(Suppl 1):S22-S23. doi: 10.1503/cmaj.180323

M uch of the previously published theories and evidence supporting patient engagement in research has focused on improving engagement in individuals with chronic disease who are participating in treatment interventions. ${ }^{1-3}$ Less is known about engaging parents as "patient partners," and very little is known about engaging parents to develop interventions designed to prevent chronic disease in young children., ${ }^{2,3}$ Our goal was to develop a pediatric prevention trial that addressed priority areas identified by parents of young children in Ontario. We designed a randomized controlled trial (RCT), the Parenting and Addressing at-Obesity Risk Early Years Intervention With Home Visits in Toronto (PARENT) trial (www.targetkids.ca/parent), to determine whether an integrated primary care and public health intervention that includes parenting-skills training and home visits can lead to improved weight status and mental health outcomes in young children.

The PARENT trial is a pragmatic, parallel-group, one-to-one, superiority RCT (ClinicalTrials.gov NCT03219697) that aims to recruit 120 children aged 18 to 36 months who are at risk of obesity. Children and their families will be recruited through TARGet Kids!, a primary care-based research network in Canada. TARGet Kids! (www.targetkids.ca) provides a platform to expand knowledge on patient engagement and involves parents as representatives for healthy young children enrolled in primary care-based prevention trials, through their mission to partner with community health care providers, families and children and create knowledge to raise healthy children. Young children in Canada and their families access primary care frequently in the first few years of life, making primary care an ideal setting to implement and test a scalable intervention aimed at preventing obesity.

The concept of engaging parents and families in the PARENT trial aligns with the principles and core beliefs of the Strategy for Patient-Oriented Research (SPOR). ${ }^{4}$ A recent scoping review identified that there is insufficient literature on engaging parents to aid with planning for a trial. ${ }^{2}$ To address this gap in the literature, we previously recruited 115 parents and 42 clinicians through the TARGet Kids! network via email and invitation letters to parents, and included those who agreed to participate in a

\section{KEY POINTS}

- Little is known about parent engagement in prevention research for young children.

- TARGet Kids! is a primary care-based research network in Canada, dedicated to parent engagement in prevention trials to promote healthy growth and development in young children.

- The Parenting and Addressing at-Obesity Risk Early Years Intervention With Home Visits in Toronto (PARENT) trial intervention comprises group-based parenting-skills training in primary care practices and home visits from public health nurses to support healthy behaviours.

- Meaningful parent involvement has resulted in improved recruitment strategies and informed our research design to enhance impact for children.

priority-setting consensus workshop. We used the James Lind Alliance framework, 5,6 a priority-setting methodology, to identify the most important unanswered research questions in pediatric preventive care from the perspectives of parents of young children and clinicians in Ontario (methods are described elsewhere ${ }^{7}$ ). Parents and clinicians attended a consensus workshop and used the nominal group technique (a type of small-group discussion to prioritize key ideas ${ }^{8}$ ); they identified important areas as research priorities, including preventing obesity, advancing parenting skills and promoting mental health. These three priorities informed the intervention, design and outcomes in the PARENT trial.

Building on this formative work undertaken by TARGet Kids!, the PARENT research team has executed an engagement plan inspired by the International Association for Public Participation framework. ${ }^{9} \mathrm{~A}$ lack of training opportunities for parent partners was identified as a key challenge in parent-engagement research. ${ }^{2}$ Before the launch of the PARENT trial, a parent partner recruited through TARGet Kids! social media and another recruited by a research coordinator, along with research team members, completed training in the research process and scientific-research literacy by attending a full-day workshop on patient engagement 
and knowledge translation run by SPOR. One parent partner (coauthor E.T.) and other research team members met regularly in the early phases of the project, in person and via telephone as necessary.

Through active engagement, our parent partners brought their perspectives on the trial design, recruitment strategies, target audience for the findings, and methods to translate and disseminate research findings in meaningful ways to the public. With their input, we developed an animated white-board video as a novel recruitment strategy; it is an efficient and informative way to help researchers to recruit parents while they are in the waiting rooms of their primary care physicians (https://youtu.be/ hIBVF_5ZQnI). Further to this work, two advisory panels of 8-10 parents, called parent panels, are being established through the TARGet Kids! PARENT trial. In collaboration with the Knowledge Translation Program (https://knowledgetranslation.net), the parent panels will provide recommendations and advice on how to improve trial design, recruitment strategies and engagement with families to inform future studies, as well as help to prioritize research outcomes and provide suggestions on ways to translate our findings effectively.

One of the main challenges we experienced was the inherent unpredictability of research processes, including the long waiting period for ethics approval that led to periods of limited communication between researchers and parent partners. As a result, some participants were concerned that parental involvement would be tokenistic, and researchers feared that parent partners would become disengaged owing to a lack of immediate results from their contributions. ${ }^{2}$ Parent partners typically volunteer their time in research, and as such they require flexibility in planning of meetings, and accommodations for meals and child care, for example. ${ }^{2}$ We learned that, in future, we should clearly and transparently outline roles, expectations and anticipated timelines from the outset, and acknowledge parent partners' time by offering honorariums.

Parents are highly invested in ensuring good health care for their children, and therefore they are highly motivated and engaged. ${ }^{2}$ Engaging parents in all aspects of the PARENT trial helped us to shift our thinking about the research. In informal discussions, parent partners emphasized that we need to be cautious in using language that may raise concerns for parents (e.g., "obesity" or "at risk of obesity") when referring to their children, especially for parents of children who may not recognize health problems related to overweight. Input from parent partners enabled us to consider issues that are important to families participating in research, including parenting skills to manage challenging behaviours and focusing on mental health outcomes.

The impact of patient and parent engagement work thus far, and our continued planned work through our parent panels, will be valuable in helping us to improve the way in which we develop, deliver and measure the impact of our interventions to fit the needs of children and their parents. High-quality research dedicated to developing the science of parent-oriented research is needed. The PARENT trial provides an opportunity to build the evidence base to support best practice guidelines to engage parents in prevention research that can ultimately improve outcomes for children and their families.

\section{References}

1. Amirav I, Vandall-Walker V, Rasiah J, et al. Patient and researcher engagement in health research: a parent's perspective. Pediatrics 2017;140:e20164127.

2. Shen S, Doyle-Thomas KAR, Beesley L, et al. How and why should we engage parents as co-researchers in health research? A scoping review of current practices. Health Expect 2017;20:543-54.

3. Domecq JP, Prutsky G, Elraiyah T, et al. Patient engagement in research: a systematic review. BMC Health Serv Res 2014;14:89.

4. Strategy for patient-oriented research - patient engagement framework. Ottawa: Canadian Institutes of Health Research; 2014. Available: www.cihr-irsc. gc.ca/e/48413.html (accessed 2018 Mar. 12).

5. Cowan K, Oliver S. The James Lind Alliance guidebook, version 5. Southampton (UK): James Lind Alliance; 2013. Available: www.jlaguidebook.org/pdfguidebook/ guidebook.pdf (accessed 2018 Mar. 12).

6. Elwyn G, Crowe S, Fenton M, et al. Identifying and prioritizing treatment uncertainties: patient and clinician engagement in the identification of research questions. J Eval Clin Pract 2010;16:627-31.

7. Lavigne M, Birken CS, Maguire JL, et al. Priority setting in paediatric preventive care research. Arch Dis Child 2017;102:748-53.

8. Harvey N, Holmes CA. Nominal group technique: an effective method for obtaining group consensus. Int J Nurs Pract 2012;18:188-94.

9. Core values for the practice of public participation. Sooke $(B C)$ : International Association for Public Participation Canada (IAP2); 2015. Available: https:// iap2canada.ca/corevalues (accessed 2018 Mar. 12).

More information on this project is available at www.ossu.ca/ IMPACTAwards.

Competing interests: None declared.

This article was solicited and has been peer reviewed.

Affiliations: Child Health Evaluative Sciences (Kim, Birken), SickKids Research Institute; parent partner (Tavares); Department of Paediatrics (Birken), Faculty of Medicine, University of Toronto, Toronto, Ont.

Contributors: All of the authors contributed to the conception and design of the work, drafted the manuscript, revised it critically for important intellectual content, gave final approval of the version to be published and agreed to be accountable for all aspects of the work.

Funding: This work was supported by OSSU (the Ontario SPOR [Strategy for Patient-Oriented Research] SUPPORT [Support for People and Patient-Oriented Research and Trials] Unit). The funding agency had no role in the design and conduct of the study, collection, management, analyses or interpretation of the results of this study or in the review of the manuscript.

Correspondence to: Catherine Birken, catherine.birken@sickkids.ca

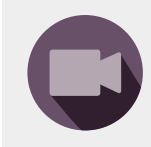
The TARGet Kids! PARENT video is available at https:// youtu.be/hIBVF_5ZQnl

The TARGet Kids! video is available at www.youtube.com/ watch?v=-8bmbztoRkw\&feature=youtu.be 